\title{
Breath sampling control for medical application
}

\author{
Wolfgang Vautz • Jörg I. Baumbach • \\ Michael Westhoff • Klaus Züchner • \\ Eike T. H. Carstens • Thorsten Perl
}

Received: 10 December 2009/Revised: 29 January 2010 /Accepted: 2 February 2010 /Published online: 20 February 2010

(C) Springer-Verlag 2010

\begin{abstract}
Sampling of breath under human control or automated control with sensors was combined with chemical determination of a synthetic sample using multicapillary column ion mobility spectrometry to measure quantitative variability. Variation was $19 \%$ with an automated inlet and $33 \%$ with human control. Sensors to operate an automated inlet were also evaluated with human subjects and included carbon dioxide $\left(\mathrm{CO}_{2}\right)$, flow (direction and velocity), volume (integrated from the flow rate) and humidity, all operating in the mainstream of exhaled air. The flow sensor provided a measure of sampling of breath from the upper airways and other sensors gauged exclusive sampling of the end-tidal volume as well. Sensors for volume and $\mathrm{CO}_{2}$ exhibited identical profiles, using appropriate threshold values, in reference to inspiration and expiration. A sensor for humidity lagged inspiration and expiration with a delay of $300 \mathrm{~ms}$ and therefore is diminished in value. The sensors recommended for an automated inlet for breath sampling are $\mathrm{CO}_{2}$ and the exhaled or tidal volume though tidal volume varies significantly with personal physiognomy. This necessitates an evaluation of a subject to establish a threshold setting
\end{abstract}

W. Vautz $(\bowtie) \cdot J$. I. Baumbach

ISAS - Institute for Analytical Sciences,

Dortmund, Germany

e-mail: vautz@isas.de

M. Westhoff

Lung Hospital,

Hemer, Germany

K. Züchner • E. T. H. Carstens · T. Perl

Department of Anaesthesiology,

Emergency and Intensive Care Medicine, University of Göttingen,

Göttingen, Germany and $\mathrm{CO}_{2}$ is the single best parameter providing the availability of sensor signal within $50 \mathrm{~ms}$.

Keywords Expiration · Inspiration · Humidity

Carbon dioxide - Tidal volume . End-tidal volume .

Breath analysis $\cdot$ Ion mobility spectrometry

\section{Introduction}

Exhaled breath is a carrier of comprehensive information about the metabolic state of humans $[1,2]$ and the collection of samples significantly affects the diagnostic value of a measurement. Usually sampling is adapted to the requirements of a measurement and to the relevant medical condition for reproducible and reliable sampling. Significantly different findings of chemical composition of breath can be expected between samples obtained from the upper airways and from those taken at the end-tidal volume. Only one of these sampling methods will be used in clinical studies, depending on the application, e.g., the upper airway volume for Halitosis studies or the end-tidal volume for human metabolite studies.

The value of ion mobility spectrometry (IMS) coupled with a rapid pre-separation (muti-capillary columns or MCC) for the analyses of human breath has been described in detail recently with examples where MCC/IMS has been applied with medical benefits [3]. The method is characterized by sensitivities down to the $\mathrm{ppt}_{\mathrm{V}}$ range and selectivity based on ionization properties of the analyses and can be made using only $8 \mathrm{~mL}$ samples of breath. Characteristic pattern for diseases can be observed as statistically valid from authentic breath samples obtained in clinical trials at the Lung Hospital in Hemer, Germany [4-7]. In other studies, serum concentrations of anaesthetics 
were determined from breath concentrations at the Department of Anaesthesiology, University of Göttingen, Germany and suggested the possibility for on-line anaesthesia control using MCC/IMS monitoring of exhaled breath [8].

In hospital studies from early developments with $\mathrm{MCC} /$ IMS, an operator controlled the sampling step and adapted the method to a subject's physical condition. In a next development, e.g. for use during anaesthesia, the patients were ventilated and the sampling was automated from concentrations of respired carbon dioxide $\left(\mathrm{CO}_{2}\right)$ [8-11]. Since these sensors are comparable expensive and slow responding with times of about $300 \mathrm{~ms}$, already within the range of a single expiration at high respiratory rates, a comprehensive investigation was merited on the suitability of parameters and sensors (including $\mathrm{CO}_{2}$, humidity, flow and volume of the exhaled breath) in breath sampling.

Since breath measurement with MCC/IMS has been successful developed, applied clinically, and adapted for sensor controlled sampling, studies and comparisons of the sampling variables was made using this analytical method; however, findings here should be transferable to other comparable breath measurement methods. These may include on-line methods of proton transfer reaction/mass spectrometry $[12,13]$ or IMR/ MS [14] and off-line methods such as gas chromatography/ mass spectrometry based on sample pre-concentration with solid phase micro-extraction or polymer trap adsorption, [15] and batch sampling with Tedlar-bags [16].

\section{Experimental details}

\section{Instrumentation}

An inlet for sampling breath using a gas switching valve and ion mobility spectrometer coupled with a multicapillary column has already been described and was used here without only minor modifications [3]. All experiments were made using a sample loop of $8 \mathrm{~mL}$ and the inlet was fitted with sensors to detect humidity, flow, and carbon dioxide $\left(\mathrm{CO}_{2}\right)$, as summarised in Table 1 .

\section{Sampling procedures}

The study with ventilated patients was approved by the ethics committee of the University of Göttingen (protocol No. 01/07/06). All patients gave written informed consent to participate in the investigation.

\section{Operator controlled sampling}

The subject breathes directly through the sample loop (see Fig. 1a). The operator chooses the portion of breath which should be analysed based on observation of the breathing
Table 1 Sensors used for the detection of the control parameters humidity, flow and carbon dioxide $\left(\mathrm{CO}_{2}\right)$

\begin{tabular}{llll}
\hline $\begin{array}{l}\text { Control } \\
\text { parameter }\end{array}$ & Sensor type & Detection limits & $\begin{array}{l}\text { Response } \\
\text { time }\end{array}$ \\
\hline Flow & ISAS-Design & $2-100 \mathrm{~L} / \mathrm{min}$ & $30 \mathrm{~ms}$ \\
Flow & $\begin{array}{l}\text { Hamilton Sensor } \\
\text { coupled to a } \\
\text { differential } \\
\text { pressure sensor }\end{array}$ & $2-100 \mathrm{~L} / \mathrm{min}$ & $50 \mathrm{~ms}$ \\
& & & \\
& 113LP02G-PCB, & & \\
& $\begin{array}{l}\text { Sensortechnics, } \\
\text { Puchheim, Germany }\end{array}$ & & \\
Humidty & heated capacitive \\
& sensor for absolute & $1 \mathrm{mg}-60 \mathrm{mg}$ & $<200 \mathrm{~ms}$ \\
humidity & $\mathrm{H}_{2} 0 / \mathrm{L}$ & \\
$\mathrm{CO}_{2}$ & $\begin{array}{l}\text { IRMA, PhaseIn, } \\
\text { Danderyd, Sweden }\end{array}$ & $0.2 \% \mathrm{~V} / \mathrm{V}$ & $300 \mathrm{~ms}$ \\
& & & (determined \\
& & & by UMG) \\
\hline
\end{tabular}

pattern of the subject-which is generally the end-tidal breath - and manually initiates the analysis. This is accomplished when the volume of the sample loop is passed into the pre-separation column (see Fig. 1c).

\section{Sensor controlled bypass sampling}

Parameters used to control sampling were monitored continuously for the flow of breath using sensors for inspired or expired air in the main stream (see Fig. 1b). The data acquisition software enabled the setting of a threshold value for these control parameters and a sample is drawn from the mainstream through the sample loop only when a threshold was exceeded. Sampling was made for a pre-determined time duration which the sample loop was flushed with sample. After the sampling period, the 6-way valve was automatically switched, thus starting the measurement (see Fig. 1c). When the volume is used as control parameter, the data from the flow sensor is integrated over time.

Comparisons of sampling by direct, operator control and by flow sensor control were made using a single subject during a few minutes interval using two MCC/IMS instruments of the same design and dedicated inlet designs. The measurements were repeated by changing the control between the $2 \mathrm{MCC} / \mathrm{IMS}$ and by varying the sequence to avoid sampling biases or artefacts. The threshold for the flow sensor was set to half of the maximum, thus avoiding significant influence of the breath from the upper airways. In operator control of sampling, the operator sought to introduce the sample only for end-tidal breath.

Laboratory studies

A humid gas volume of air was prepared using 2-nonanone as a synthetic sample to probe sampling efficiency with an 
Fig. 1 Scheme of the sampling system used for the present study. The 6-way valve may be switched manually for operated controlled sampling as well as automatically for sensor controlled sampling. a) The subject exhales directly through the sample loop. b) The subject exhales through a mouthpiece and the sensor signal is used to decide when a sample is drawn by help of a pump at (4) from the mainstream through the sample loop. c) After switching the 6-way valve from "sampling" to "sample introduction", the volume of the sample loop is introduced into the MCC at (1) and after pre-separation into the IMS by help of the carrier gas at (1)
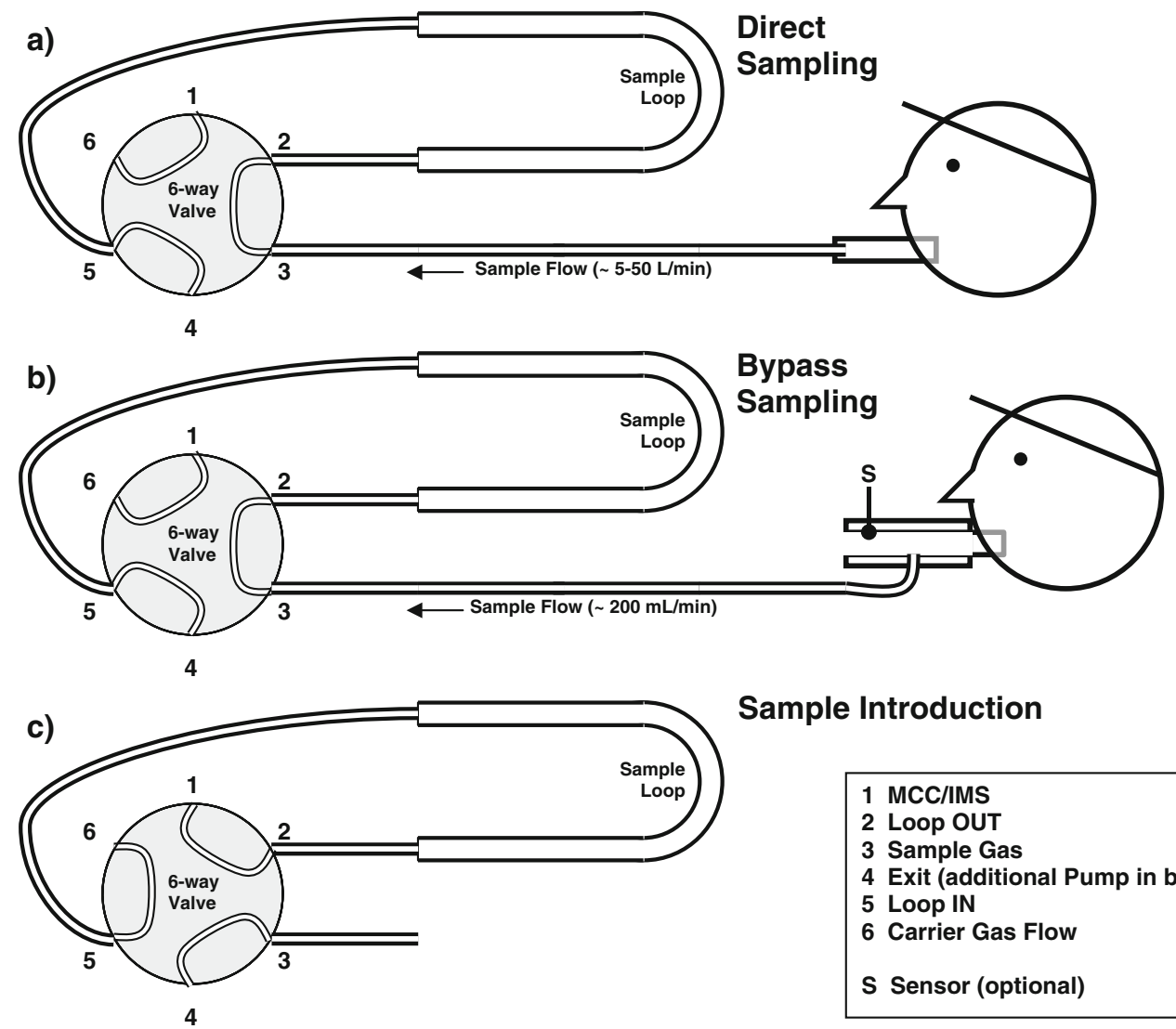

Sample Introduction

1 MCC/IMS
2 Loop OUT
3 Sample Gas
4 Exit (additional Pump in b)
5 Loop IN
6 Carrier Gas Flow
5 Sensor (optional)

uncomplicated measurement using a proved calibration gas generator (HovaCAL 3834SC VOC, Inspire Analytical Systems GmbH, Frankfurt am Main, Germany, see [17]).

\section{Results and discussion}

Results using a humidified vapour sample with 2nonanone to simulate breath in controlled concentrations provided a comparison of both sampling methods (see Fig. 2) with a chemically simple and stable sample. While response was observed with both methods of sampling and MCC-IMS, results were qualitatively identical, showing no influence of the sampling on retention time or drift time. However, strong variations in signal height or intensity of the response to 2-nonanone could be observed. A quantitative comparison of measurements from each sampling method is shown in Fig. 3 where the sensor controlled sampling resulted in higher response and better reproducibility than from manual sampling. The normalised concentrations showed a standard deviation of $66 \%$ for operator controlled sampling compared to $13 \%$ from flow controlled bypass sampling. Since both studies were operated under stable conditions by a single individual, the variations may be attributed to variations in manually sampling only, specifically with variation in the start of sampling. Though manual sampling is significantly worse than automated sampling, variations still greater may result in routine clinical applications where various analysts could introduce errors in judging a subjects physical condition and the moment to stop sample collection. As a consequence, a sensor controlled sampling should be used when quantitative and medically defensible results are desired.

The response of sensors and parameters for monitoring respiration are shown in Fig. 4 where flow and humidity were sufficiently fast for real-time control of sampling, fitting close within the ranges of physiologic activity. The sensor for $\mathrm{CO}_{2}$ exhibited a response time of $300 \mathrm{~ms}$ and the signal shown in Fig. 4 was adjusted in time scale so comparisons can be made in slope and shapes of response to the other parameters. This type of delay is typical and sufficient for most of the available capnographic sensors since in general this concentration is not used to control any other analytical or sampling procedure during breathing; however, this sensor can be used only for sampling when the breathing frequency is $<2 \mathrm{~s}$. For higher frequency as quite usual for ventilated patients, other and faster sensors - at least few of such sensors are available - are necessary for an automated sampling inlet. The slope of all 4 parameters is presented in Fig. 4 and several conclusions can be made from comparisons of response patterns: 

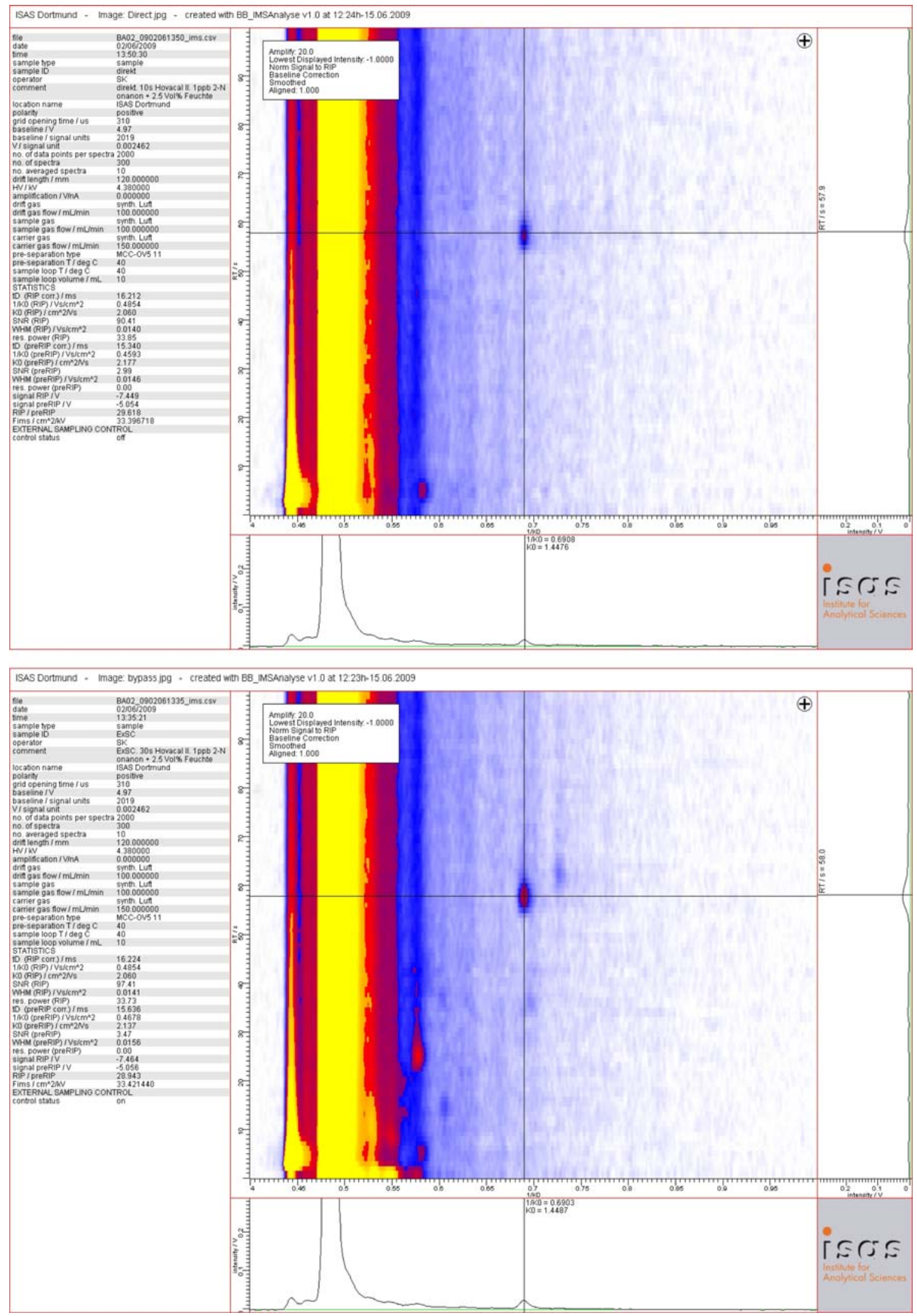

Fig. 2 MCC/IMS chromatogram of the synthetic breath sample, of 2-nonanone in humid air, (indicated with the cross line) derived from direct, operator controlled sampling (top) and from flow controlled bypass sampling (bottom) 


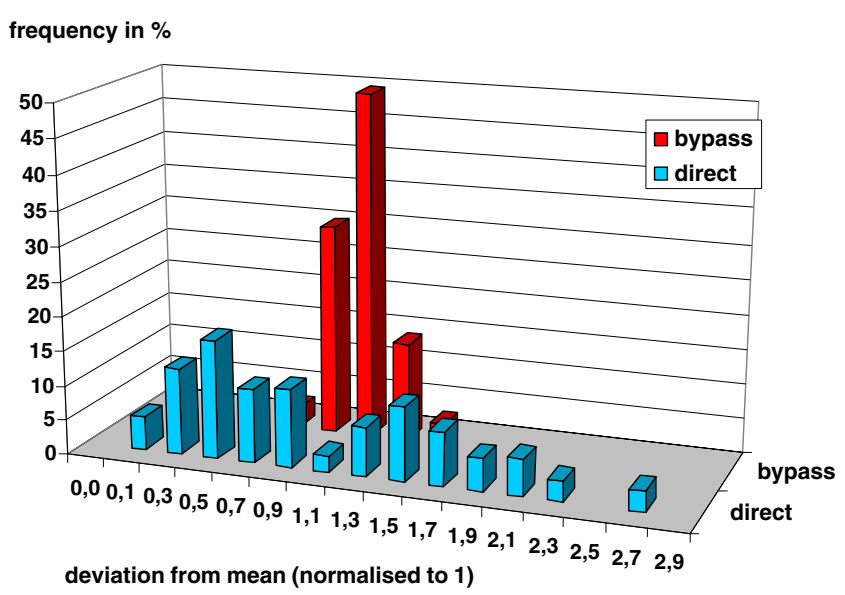

Fig. 3 Comparison of quantitative MCC/IMS response for 2nonanone determination in humid air by direct sampling with an operator and with an automated sensor controlled bypass sampling using a flow sensor in this case and a constant concentration of $1 \mathrm{ppb}_{\mathrm{V}}$ provided by HovaCAL

Tidal flow

Using a unique threshold limit for the flow, expiration and inspiration can be differentiated, though as can be seen in Fig. 4, this will always lead to a predominant sampling of the air of the upper airways as the flow follows a steep increase followed by a slowly decrease. If the threshold is set to zero, the entire tidal volume will be sampled and will lead to a mixture of the end-tidal and upper airway breath. This could be solved by applying two different threshold limits for sampling "on" and "off". Setting both of them in the decreasing slope of the curve would best enable endtidal sampling; however, this requires elaborate programming of the data acquisition software and furthermore, a calibration to the particular subject is required in all cases.

\section{Humidity}

The humidity signal rises during expiration and thus enables a differentiation of the breath from the upper airways from the end-tidal volume. A tailing of response on the decrease during inspiration is significant and slow resulting in the collection of an increasing partition of inspiration air for lower threshold limits.

\section{$\mathrm{CO}_{2}$}

This parameter is an appropriate measure of the origin of the samples air as the concentration is almost independent on all other parameters such as tidal flow and from the background level as well. The slope is repeatable and the changes rapidly follow the tidal flow. Thus, a clear distinction of end-tidal breath and air from the upper airways is possible and enables the exclusive sampling of both partitions of breath.

\section{Tidal volume}

Tidal volume can be derived online from integration of flow rates and with suitable thresholds limits, the switching of sampling "on" and "off" enables an exclusive sampling of end-tidal breath as well. This is almost identical to the use of $\mathrm{CO}_{2}$. However, calibration for each particular subject is needed for tidal volume as significant physical differences may lead to different tidal flow and volume.

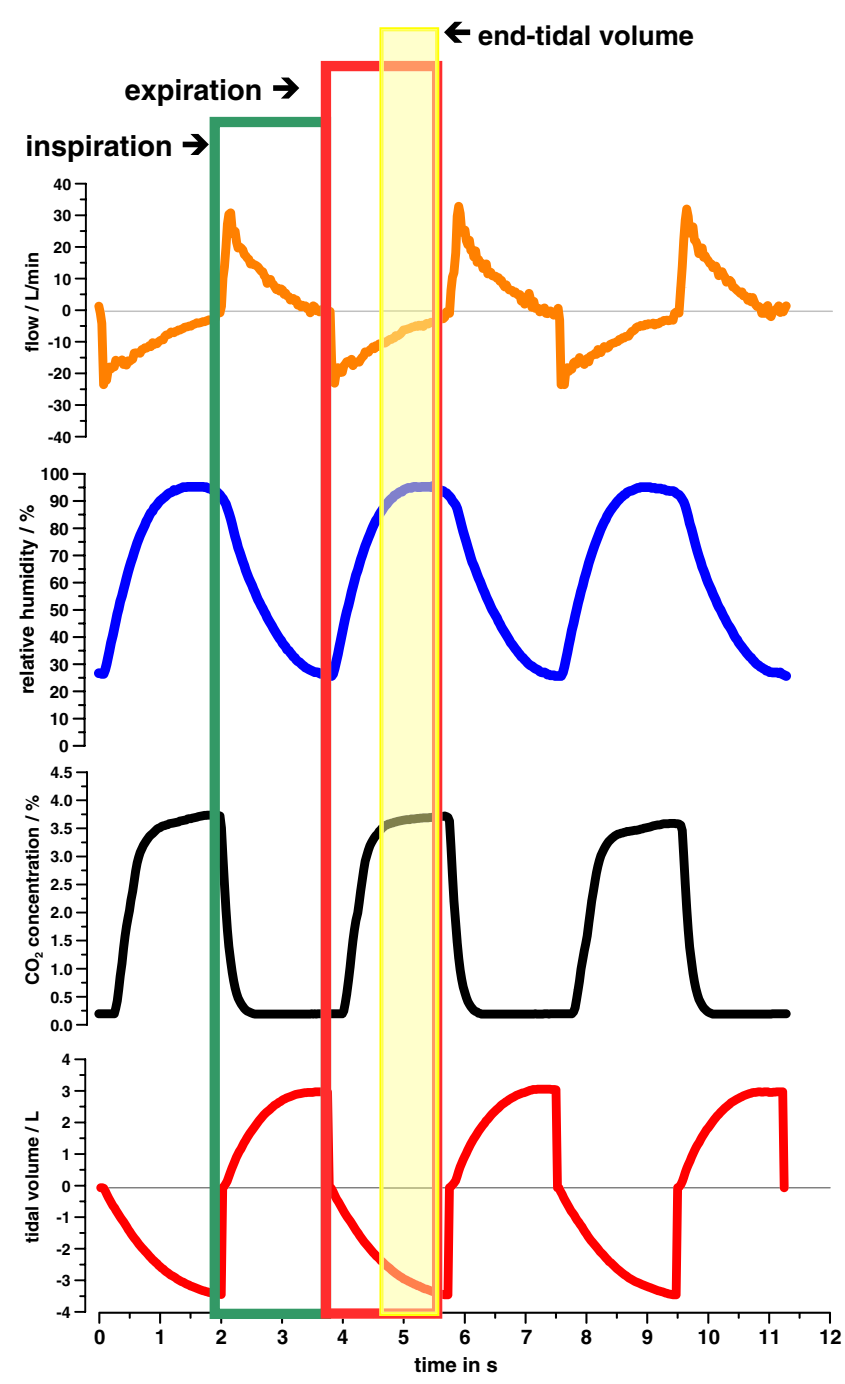

Fig. 4 Comparison of the control parameters and of the influence of threshold values in the breath of a ventilated patient as an example. Inspiration, expiration and the end - tidal volume are indicated. The response delay of the $\mathrm{CO}_{2}$ sensor was corrected 


\section{Conclusions}

Approaches for human breath sampling through direct, operator controlled sampling and through sensor controlled bypass sampling showed that sensor controlled bypass sampling with a flow sensor exhibited higher reproducibility than the direct operator controlled sampling. Direct, operator controlled sampling showed detection of pattern of metabolites, as with the sensor control and only the quantitative performance was degraded.

A comparison of the simultaneously determined slope of control parameters humidity, tidal flow, tidal volume and $\mathrm{CO}_{2}$ concentration - all measured in human breathshowed that a flow sensor can be applied optimally for the sampling of breath from the upper airways only. During expiration, the slope follows a steep increase in the beginning followed by a more or less exponential decrease. Therefore, with a relatively high threshold value, the first part of the volume of the exhaled breath will be sampled.

In contrast, for analysis of end-tidal breath, the control using the volume or $\mathrm{CO}_{2}$ as parameters is required. The concentration in end-tidal breath ends up a plateau and can therefore easily be distinguished by the sensor from the first part of breath. However, using appropriate threshold values, the tidal volume - obtained from integration of the tidal flow-and $\mathrm{CO}_{2}$ obtain identical results.

The humidity follows the slope of inspiration and expiration with a delay and therefore is less suitable.

As a consequence, $\mathrm{CO}_{2}$ and the expired volume are the most suitable control parameters for end-tidal breath sampling with $\mathrm{CO}_{2}$ being the most appropriate parameter if the sensor signal is available after less then $50 \mathrm{~ms}$ as it is independent on the subjects physiognomy. Indeed, this is also the most expensive solution compared to expired volume.

Due to the response time of $300 \mathrm{~ms}$ the $\mathrm{CO}_{2}$ sensor used in the present study is not suitable for the breath analysis using MCC/IMS in all situations. Depending on the physical conditions or - in case of mechanical ventilation on the setup of the ventilator - the breathing frequency can rise easily to more than $1 \mathrm{~Hz}$. Then the duration of expiration is less than $500 \mathrm{~ms}$ and cannot be resolved properly by a sensor with $300 \mathrm{~ms}$. Such sensors with a response time in the range of $50 \mathrm{~ms}$ are not widely spread, however, some of such sensors are in fact available. Although all results were obtained using a MCC/IMS instrument, finding should be useful for other on-line analytical methods and for off-line sampling as well.

Acknowledgements The financial support of the Bundesministerium für Bildung und Forschung and the Ministerium für Wissenschaft und Forschung des Landes Nordrhein-Westfalen, the high-tech strategy funds of the Federal Republic of Germany (Project Metabolit-
01SF0716) and of the European Union (Project EU-SGL) is gratefully acknowledged. The dedicated work of the stuff at ISAS - Institute for Analytical Sciences, the Lung Hospital in Hemer and at the Department of Anaesthesiology, Emergency and Intensive Care Medicine at the University of Göttingen was indispensable for the successful investigations.

\section{References}

1. Risby TH, Solga SF (2006) Current status of clinical breath analysis. Appl Phys B Lasers Opt 85:421-426

2. Zolotov YA (2005) Breath analysis. J Anal Chem 60:497

3. Vautz W, Nolte J, Fobbe R, Baumbach JI (2009) Breath analysisperformance and potential of ion mobility spectrometry. J Breath Res 3, 036004 (8pp) doi:10.1088/1752-7155/3/3/036004

4. Westhoff M (2005) Ion mobility spectrometry: a new method for the detection of lung cancer and airway infection in exhaled airfirst results of a pilot study. Chest 128:155S

5. Baumbach JI, Westhoff M (2006) Ion mobility spectrometry to detect lung cancer and airway infections. Spectrosc Eur 18:22-27

6. Westhoff M, Litterst P, Freitag L, Baumbach JI (2007) Ion mobility spectrometry in the diagnosis of sarcoidosis results of a feasibility study. J Physiol Pharmacol 58:739-751

7. Westhoff M (2008) Ion mobility spectrometry for the detection of volatile organic compounds in exhaled breath of lung cancer patients—results of a pilot study. Thorax 64: 2008

8. Perl T, Carstens ETH, Hirn A, Quintel M, Vautz W, Nolte J, Jünger M (2009) Determination of serum propofol concentrations by breath analysis using ion mobility spectrometry. $\mathrm{Br} \mathrm{J}$ Anaesthesia 103(6):822-827

9. Schubert J, Spittler KH, Braun G, Geiger K, Guttmann J (2001) $\mathrm{CO}_{2}$-controlled sampling of alveolar gas in mechanically ventilated patients. Appl Physiol 90:486-492

10. Birken T, Schubert J, Miekisch W, Nöldge-Schomburg G (2006) A novel visually $\mathrm{CO} 2$ controlled alveolar breath sampling technique. Technol Health Care 14(6):499-506

11. Dolch ME, Frey L, Hornuss C, Schmoelz M, Praun S, Villinger, Schelling G (2008) Molecular breath-gas analysis by online mass spectrometry in mechanically ventilated patients: a new softwarebased method of CO2-controlled alveolar gas monitoring.J. Breath Res 2: doi:10.1088/1752-7155/2/3/037010

12. Harrison GR, Critchley AD, Mayhew CA, Thompson JM (2003) Real-time breath monitoring of propofol and its volatile metabolites during surgery using a novel mass spectrometric technique: a feasibility study. Br J Anaesth 91:797-799

13. Herbig J, Müller M, Schallhart S, Titzmann T, Graus M, Hansl A (2009) On-line breath analysis with PTR-TOF. J Breath Research 3: doi:10.1088/1752-7155/3/2/027004

14. Hornuss C, Praun S, Villinger J, Dornauer A, Moehnle P, Dolch M, Weninger E, Chouker A, Feil C, Briegel J, Thiel M, Schelling G (2007) Real-time monitoring of propofol in expired air in humans undergoing total intravenous anesthesia. Anesthesiology 106:665-674

15. Zimmermann D, Hartmann M, Moyer MP, Nolte J, Baumbach JI (2007) Determination of volatile products of human colon cell line metabolism by GC/MS analysis. Metabolomics 3:13-17

16. Amann A, Schmid A, Scholl-Buergi S, Telser S, Hinterhuber H (2005) Breath analysis for medical diagnosis and therapeutic monitoring. Spectrosc Eur 17:18-20

17. Vautz W, Schmäh M (2009) HovaCAL ${ }^{\circledR} — a$ generator for multicomponent humid calibration gases. Int J Ion Mobil Spec 12:139147 